\author{
Yang GENG, PhD, Corresponding Author \\ Email: ygeng1989@163.com \\ College of Economics and Management \\ Nanjing Forestry University \\ Professor Yu-lin ZHANG, PhD \\ Email: zhangyl@seu.edu.cn \\ School of Economics and Management \\ Southeast University
}

\title{
PRICING IN TWO-SIDED MARKETS WITH DYNAMIC SUBSCRIPTION PROCESS AND USERS' EXPECTATIONS
}

\begin{abstract}
In a dynamic framework, we study pricing strategies in two-sided markets while considering users' expectations. The results show that in the steady-state equilibrium, except for the indirect network effects, the growth rates and users' beliefs may also cause skewed pricing. In non-equilibrium, we find that in the early period, the platform may delay the entrance of users on one side and attract only users on the other side if the costs of attracting a large user base on this side are large. Besides, when the weight coefficients of the conjectured numbers that depend on users' beliefs are high, the optimal price trajectories increase rapidly in the period when the numbers of users are low and then converge to low equilibrium prices.
\end{abstract}

Keywords: network effects; dynamic pricing; two-sided markets; expectations.

\section{JEL Classification: C61}

\section{Introduction}

In recent years, the markets in which two or more sides of users interact through platforms have attracted the attention of both business and academia. Examples include online shopping markets such as Amazon or eBay, video games such as Xbox 360, payment systems such as MasterCard or Visa, etc. A major feature of these two-sided (or multi-sided) markets (Weyl, 2010; Armstrong, 2006) is indirect network effects between distinct sides of users. It means that the benefits of a user on one side are influenced by the number of users on the other side. For instance, buyers are more willing to subscribe to an online shopping platform with many sellers because they can find more suitable products. On the other side, with more buyers on the platform, subscribed sellers can sell more products.

In such markets, pricing strategies are important. Early researchers have found that in equilibrium, skewed pricing can be profitable (Rochet and Tirole, 2003;

DOI: $10.24818 / 18423264 / 55.3 .21 .09$ 
Armstrong, 2006). For example, on an online shopping platform, such as Amazon, buyers can use the platform for free in most cases, while sellers need to pay for selling goods, such as subscription fees or commission fees. By implementing this pricing strategy, a platform in the markets may make a loss on the side where users are subsidized. However, with the indirect network effects, it can earn money from users on the other side. In addition, except for studies in a static framework focusing on equilibrium prices, intertemporal pricing strategies are also used by many two-sided platforms. Take Uber as an example. When Uber entered the market, it spent heavily on subsidizing passengers and drivers. However, with enough subscribed users, it began to reduce subsidies and raise prices.

In this paper, we focus on dynamic pricing in two-sided markets. In a dynamic framework, users may hold expectations because the benefits that they can make in the future are unknown. Users may base expectations on participations in history (Hałaburda et al., 2020) or beliefs (Jullien and Pavan, 2019). When users base expectations on participations in history only, we say that these users are myopic and the expectations are always fulfilled (Evans and Schmalensee, 2010). However, if users on one side form beliefs regarding participations of the other side in the future, the conjectured number that depends on users' beliefs may differ from the existing number, which implies that the expectations may not be fulfilled in non-equilibrium.

To address this question, we build a dynamic model to describe the dynamic subscription process of users. In the dynamic process, users base their subscription decisions not only on the existing number of users on the other side (the effect of history) but also on the existing number of users on the same side (the effect of beliefs). By using the optimal control theory, we find that the expectation bias that comes from the difference between beliefs and reality affects the optimal prices in non-equilibrium. However, it does not affect the equilibrium prices since users are rational. Hence, in the long term, the real status of the platform, such as the service quality and the numbers of users, is the key to make profits. In the equilibrium of the dynamic model, we also find that except for the indirect network effects, the growth rates and users' beliefs may also give rise to skewed pricing in two-sided markets. In non-equilibrium, the results show that in the early period, the platform may delay the entrance of users on one side and attract only users on the other side if the costs of attracting a large user base on this side are large.

The rest of the paper is organized as follows. The related literature is reviewed in the next section. We then set forth our model in Section 3. In Section 4, we theoretically analyze dynamic pricing strategies and in Section 5, we provide an equilibrium analysis. The optimal trajectory is discussed through numerical analysis in Section 6. Section 7 concludes the paper.

\section{Literature review}

Our research primarily relates to two streams of research. The first related stream involves studies on two-sided markets. In this research area, a large body of research on pricing problems has been conducted under a static framework. Rochet and Tirole (2003), for example, analyze two-sided platforms that primarily charge transaction fees and find that in a monopoly platform, the optimal price structure is 
Pricing in Two-Sided Markets with Dynamic Subscription Process and Users' Expectations

decided by the ratio of price elasticities on the two sides. Armstrong's (2006) results show that the optimal subscription fees are also affected by cross-group externalities compared with the standard Lerner formula, which implies that the platform can set a low price on one side and benefit from users on the other side. Our study differs from theirs in that we focus on price trajectory in a dynamic framework.

In addition, under incomplete information, expectations are considered by some researchers who study markets with network effects following from Katz and Shapiro (1985) who study markets with direct network effects to recent research on two-sided markets, such as Caillaud and Jullien's (2003), Gabszewisz and Wauthy (2014), Hagiu and Hałaburda (2014) and Belleflamme and Peitz (2019). In these papers, the expected numbers are exogenously given, but will be equal to the real numbers in the fulfilled expectations equilibrium (see Katz and Shapiro, 1985). However, they do not consider the effect of history on expectations (Hałaburda et al., 2020) and users' beliefs (Jullien and Pavan, 2019; Hałaburda and Yehezkel, 2019; Markovich and Yehezkel, 2019). In this paper, because we study pricing problem in a dynamic framework, we assume that expectations are endogenously decided by participations in history and users' beliefs.

The second stream is about dynamics in markets with network effects. A famous question in this stream is the startup problem. Specifically, due to the network effects, attracting users in the initial period becomes difficult. In markets with direct network effects, Rohlfs's (1974) results show that multiple equilibria may exist, and when the number of users is higher than the critical mass (one of the equilibria), the number of users can grow by itself. In two-sided markets, Evans and Schmalensee (2010) study the same problem and find a unique critical trajectory. Similarly, their results show that the markets can reach the equilibrium with high numbers of users when the initial numbers of users are above the critical trajectory. In this paper, we use their model to describe the dynamic subscription process of users. The difference is that in our model, users' beliefs can affect their subscription decisions.

Considering platforms endogenously decide prices in different periods, some researchers analyze dynamic pricing in markets with network effects. In markets with direct network effects, Dhebar and Oren $(1985,1986)$ find that the optimal price trajectory increases with the number of users and that, to ensure the number of users can grow by itself, the platform may charge lower prices than interior solutions in the initial period. Cabral's (2011) results show that if considering platforms' future pay-offs, the optimal price may not increase with the number of users. In two-sided markets, Chen and Tse (2008) discuss platform competition with linear diffusion dynamics and find that under the open-loop pure strategy, in non-equilibrium, the optimal price trajectories in the stronger platform increase with time, while in the weaker platform, the optimal price trajectory on each side first increases and then decreases. Cabral (2019) finds that the distribution of platform size at time $t$ in many cases is bimodal. 
Yang Geng, Yu-Lin Zhang

\section{Model}

For concreteness in what follows, users on the two sides are sellers (superscript s) and buyers (superscript b). Prices at time $t$ charged by the platform are $p^{b}(t)$ and $p^{s}(t)$. Let $x(t)$ and $y(t)$, respectively, denote the number of buyers and sellers on the platform at time $t$. Moreover, if the meaning is clear, we omit the argument $t$. For example, $p^{b}(t)$ will be expressed simply as $p^{b}$.

\subsection{The willingness-to-pay functions}

On the buyers' side, we use a concave willingness-to-pay function $W^{b}\left(\xi, y^{e}\right)$ to describe buyer $\xi$ 's reservation price for subscribing to the platform, where $\xi$ is uniformly distributed on $[0,1]$ and $y^{e}$ represents this buyer's expectations for the number of sellers. In this function, given $y^{e}$, a buyer with a small $\xi$ benefits more from subscribing to the platform, i.e., $W_{1}^{b}<0$, and the indirect network effects are positive, i.e., $W_{2}^{b}>0 .{ }^{1}$ In addition, to avoid the discussion of corner solutions, we assume that $W^{b}\left(1, y^{e}\right)=0$. We also consider that a buyer with a small $\xi$ benefits more from indirect network effects, i.e., $W_{12}^{b} \leq 0$. This assumption is commonly seen in the research of two-sided markets, such as Rochet and Tirole (2003) with $W_{12}^{b}<0$ and Armstrong (2006.) with $W_{12}^{b}=0$. Take online trading markets as an example. $\xi$ can correspond to an income level that is exceeded by $\xi \in[0,1]$ of the total number of buyers. Then the income of a buyer with a small $\xi$ is high, and generally high-income buyers can buy more products than low-income buyers.

On the sellers' side, $W^{s}\left(\eta, x^{e}\right)$ denotes the willingness-to-pay function of seller $\eta$, which is concave in $\left(\eta, x^{e}\right)$. In the function, $\eta$ is uniformly distributed on $[0,1]$ and $x^{e}$ represents seller $\eta$ 's expectations for the number of buyers. Similarly, on this side, we assume that $W_{1}^{s}<0, W_{2}^{s}>0, W^{s}\left(1, x^{e}\right)=0$ and $W_{12}^{s} \leq 0$.

\subsection{Users' expectations}

To simplify our analysis, we make assumptions about expectations based on a coordination device (Hałaburda and Yehezkel, 2019), where users coordinate beliefs regarding other users' subscription decisions. In the initial state, we assume that

(A0) Initial subscribers are those with low indexes $(\xi \leq x(0)$ and $\eta \leq y(0))$.

The above assumption can be explained by the fact that users with low

\footnotetext{
${ }^{1}$ In this paper, subscripts 1 and 2 of a function denote partial derivatives with respect to the first term and the second term in the bracket, respectively.
} 
Pricing in Two-Sided Markets with Dynamic Subscription Process and Users' Expectations

indexes benefit more from subscribing to the platform and then have a motive to subscribe early. For any time $t$, we assume that

(A1) A subscribed seller $\tilde{\eta}$ believes that if there exist sellers with $\eta>\tilde{\eta}$, he can remain on the platform as well. Similarly, a subscribed buyer $\tilde{\xi}$ remains on the platform if there are subscribed buyers with higher indexes.

(A2) An unsubscribed seller $\hat{\eta}$ will stay out of the market if unsubscribed sellers with lower indexes $(\eta<\hat{\eta})$ exist. Similarly, an unsubscribed buyer $\hat{\xi}$ stays out of the market if there are unsubscribed buyers with lower indexes.

(A0-A2) can be explained by the fact that users with high $\xi$ or $\eta$ benefit less from subscribing and then have less tolerance for risk. Therefore an unsubscribed seller with a small $\hat{\eta}$ is very cautious, and he will be a watcher until the number of sellers on the platform grows to his bottom line, i.e., $y(t)=\hat{\eta}$.

For marginal users, because there are no referents, they have to make decisions on their own. We assume that

(A3) Given the state at time $t: \Omega(t)=\left\{p^{b}(t), p^{s}(t), x(t), y(t)\right\}$, marginal seller $\bar{\eta}$ subscribes to the platform if and only if $W^{s}\left(\bar{\eta}, x^{e}(\bar{\eta}, \beta, \Omega)\right)>p^{s}$, where the marginal seller's expectations for the number of buyers $\left(x^{e}(\bar{\eta}, \beta, \Omega)\right)$ are a weighted average of the conjectured number of buyers $\left(x^{e}(\bar{\eta}, 1, \Omega)\right)$ and the existing number of buyers $(x): x^{e}(\bar{\eta}, \beta, \Omega)=\beta x^{e}(\bar{\eta}, 1, \Omega)+(1-\beta) x$. The conjectured number of buyers is based on the marginal seller's belief and $\beta$ is a weight coefficient for $x^{e}(\bar{\eta}, 1, \Omega)$. Since the change of the number of buyers in the future is affected by the existing number of sellers, the marginal seller concludes that the number of buyers wishing to subscribe will be $x^{e}(\bar{\eta}, 1, \Omega)=\max \left(x^{e 1}, 0\right)$, where $x^{e 1}$ satisfies $W^{b}\left(x^{e 1}, y\right)=p^{b}$. Marginal buyer $\bar{\xi}$ subscribes to the platform if and only if $W^{b}\left(\bar{\xi}, y^{e}(\bar{\xi}, \alpha, \Omega)\right)>p^{b}$, where $y^{e}(\bar{\xi}, \alpha, \Omega)=\alpha y^{e}(\bar{\xi}, 1, \Omega)+(1-\alpha) y$. Similarly, the conjectured number of sellers $\left(y^{e}(\bar{\xi}, 1, \Omega)\right)$ satisfies $y^{e}(\bar{\xi}, 1, \Omega)=\max \left(y^{e 1}, 0\right)$, where $W^{s}\left(y^{e l}, x\right)=p^{s}$

In general, (A0-A3) shows that the number of subscribers on one side increases (resp., decreases) if and only if the marginal user on this side enters (resp., leave) the markets. In contrast to studies assuming expectations are based on participations in history, we also incorporate the effect of the number of users on the same side. Such effect is commonly seen in real life. For example, in online trading markets, with group psychology or the recommendation of subscribed buyers, unsubscribed buyers usually subscribe to a platform with many subscribed buyers. On the other side, the reason why sellers base expectations on the same side may be due to some favorable factors, such as aggregation effects. 


\subsection{Demand functions}

We first study the properties of marginal users' willingness-to-pay functions. Due to the symmetric of two sides, we take the buyers' side as an example.

Lemma 1. $W^{b}\left(\bar{\xi}, y^{e}(\bar{\xi}, \alpha, \Omega)\right)$ is a concave function on $\left\{\bar{\xi}: y^{e}(\bar{\xi}, 1, \Omega)>0\right\}$, and a non-increasing function on $\left\{\bar{\xi}: \alpha y^{e}(\bar{\xi}, 1, \Omega)=0\right\}$.

Proof. All proofs appear in the Appendix.

Lemma 1 shows that $W^{b}\left(\bar{\xi}, y^{e}(\bar{\xi}, \alpha, \Omega)\right)$ may increase with $\bar{\xi}$ if expectations are partly based on beliefs (see cases with $\alpha \neq 0$ in Fig. 1). The reason is that for marginal buyers with low indexes in the early period, the numbers of subscribed buyers are small and then the conjectured number of sellers is small as well.

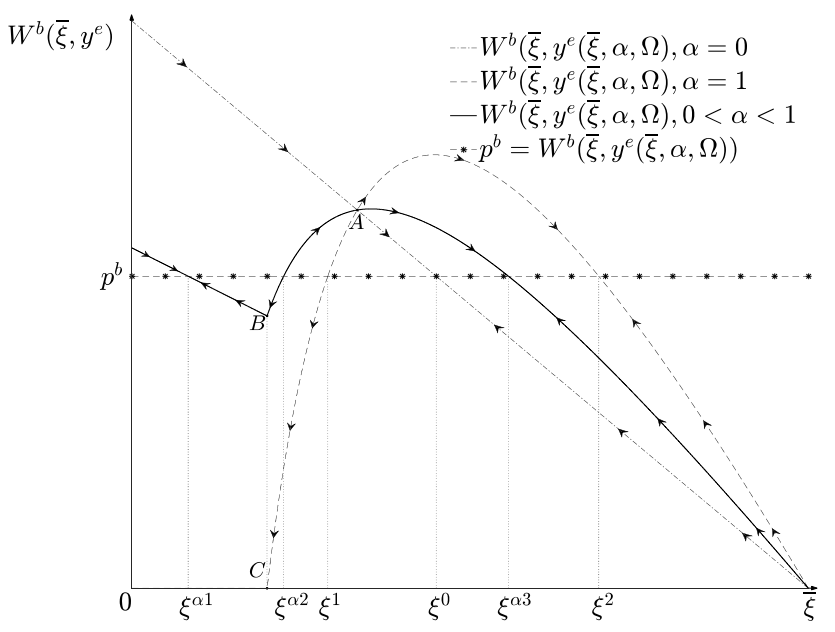

Figure 1. The willingness-to-pay curve of marginal buyers

With the analysis in Section 3.2, we then can obtain the dynamic subscription process of users, i.e., the directions of arrows in Fig. 1 and also the demand function of buyers denoted by $d^{b}(\alpha, \Omega)$ as follows: (1) If $\alpha=0$, only one equilibrium exists and $d^{b}(0, \Omega)=\xi^{0}$; (2) If $\alpha=1$, two stable equilibria exist, and $d^{b}(1, \Omega)=\xi^{2}$ when $x>\xi^{1}$, while $d^{b}(1, \Omega)=0$ when $x<\xi^{1}$; (3) If $0<\alpha<1$, there are two stable equilibria and $d^{b}(\alpha, \Omega)=\xi^{\alpha 3}$ when $x>\xi^{\alpha 2}$, while $d^{b}(\alpha, \Omega)=\xi^{\alpha 1}$ when $x<\xi^{\alpha 2}$.

\subsection{Equilibria}

In the dynamic subscription process, we assume, on the buyers' side, that $\dot{x}=d x / d t$ and is in direct proportion to the unsubscribed buyers willing to 
Pricing in Two-Sided Markets with Dynamic Subscription Process and Users' Expectations

subscribe, i.e., $\quad \dot{x}=G^{b}\left(d^{b}(\alpha, \Omega)-x\right) \quad$, where $\quad G^{b^{\prime}}=\partial G^{b} / \partial\left(d^{b}-x\right) \geq 0 \quad$ and $G^{b}(0)=0$.

On the sellers' side, we assume $\dot{y}=d y / d t=G^{s}\left(d^{s}(\beta, \Omega)-y\right)$, where $G^{s}(0)=0, \quad G^{s^{\prime}}=\partial G^{s} / \partial\left(d^{s}-y\right) \geq 0$ and $d^{s}(\beta, \Omega)$ is the demand function of sellers.

In the above diffusion model, the dynamic process stops at $\dot{x}=0$ and $\dot{y}=0$. In equilibrium, we have $d^{b}(\alpha, \Omega)=x$ and $d^{s}(\beta, \Omega)=y$. However because users' expectations are partly based on beliefs, the expected numbers may not be fulfilled. Therefore the equilibrium should also satisfy $x^{e}(\bar{\eta}, \beta, \Omega)=x$ and $y^{e}(\bar{\xi}, \alpha, \Omega)=y$.

Lemma 2. The state $\Omega=\left\{p^{b}, p^{s}, x, y\right\}$ is an equilibrium if and only if

$$
W^{b}(x, y)=p^{b} ; \quad W^{s}(y, x)=p^{s} .
$$

Lemma 2 implies that even if the subscription decisions of users are affected by their beliefs, the final equilibria are fixed with given prices.

\section{Optimal dynamic pricing strategies}

The profit function of the monopolistic two-sided platform is

$$
\Pi=\int_{0}^{+\infty} e^{-\delta t}\left(p^{b}(t) x(t)+p^{s}(t) y(t)-c(x(t), y(t))\right) d t,
$$

where $c(x(t), y(t))$ is the cost function of the platform and $\delta$ is the discount rate.

The optimization problem then is:

$$
\max \Pi=\int_{0}^{+\infty} e^{-\delta t} \pi(t, \Omega) d t,
$$

subject to $\dot{x}=G^{b}\left(d^{b}(\alpha, \Omega)-x\right), \quad \dot{y}=G^{s}\left(d^{s}(\beta, \Omega)-y\right), \quad x(0)=x^{0}$ and $y(0)=y^{0}$, where $\pi(t, \Omega)=p^{b} x+p^{s} y-c(x, y)$.

In the problem, the prices are not required to satisfy the nonnegative conditions as subsidizing users is common in two-sided markets. We apply the optimal control theory to solve this problem. The Hamiltonian function is

$$
H=e^{-\delta t} \pi(t, \Omega)+\lambda^{1} G^{b}\left(d^{b}(\alpha, \Omega)-x\right)+\lambda^{2} G^{s}\left(d^{s}(\beta, \Omega)-y\right),
$$

where $\lambda^{1}$ and $\lambda^{2}$ are the costate variables, and the current value Hamiltonian is

$$
H^{c}=\pi(t, \Omega)+\theta^{1} G^{b}\left(d^{b}(\alpha, \Omega)-x\right)+\theta^{2} G^{s}\left(d^{s}(\beta, \Omega)-y\right),
$$

where $\theta^{1}=e^{\delta t} \lambda^{1}$ and $\theta^{2}=e^{\delta t} \lambda^{2}$.

Due to the maximum principle, the optimal trajectory $\left(\tilde{p}^{b}(t), \tilde{p}^{s}(t), \tilde{x}(t), \tilde{y}(t)\right)$ should satisfy the first order conditions

$$
H_{p^{b}}^{c}=H_{p^{s}}^{c}=0
$$

and the costate equations

$$
\dot{\theta}^{1}=-H_{x}^{c}+\delta \theta^{1} ; \quad \dot{\theta}^{2}=-H_{y}^{c}+\delta \theta^{2} .
$$

As is customary, in what follows, subscripts denote partial derivatives, e.g., 
Yang Geng, Yu-Lin Zhang

$H_{p^{b}}^{c}=\partial H^{c} / \partial p^{b}, H_{p^{s}}^{c}=\partial H^{c} / \partial p^{s}$. In addition, since the network grows to a steady-state equilibrium, the optimal price trajectory should satisfy the transversality condition: $\lim _{t \rightarrow+\infty} H=0$, and the costate variables should satisfy $\lim _{t \rightarrow+\infty} \lambda^{1}=\lim _{t \rightarrow+\infty} \lambda^{2}=0$.

In the maximum principle, from Equations (1), we obtain

$$
x+\theta^{1} G^{b^{\prime}} d_{p^{b}}^{b}+\theta^{2} G^{s^{\prime}} d_{p^{b}}^{s}=0 ; y+\theta^{1} G^{b^{\prime}} d_{p^{s}}^{b}+\theta^{2} G^{s^{\prime}} d_{p^{s}}^{s}=0
$$

Due to the analysis in Section 3.2 and 3.3, the demand functions are decided by the identical equations as follows:

$$
W^{b e}=W^{b}\left(d^{b}(\alpha, \Omega), \alpha y^{e}\left(d^{b}(\alpha, \Omega), 1, \Omega\right)+(1-\alpha) y\right)=p^{b}
$$

and

$$
W^{s e}=W^{s}\left(d^{s}(\beta, \Omega), \beta x^{e}\left(d^{s}(\beta, \Omega), 1, \Omega\right)+(1-\beta) x\right)=p^{s},
$$

where $x^{e}\left(d^{s}(\beta, \Omega), 1, \Omega\right)$ and $y^{e}\left(d^{b}(\alpha, \Omega), 1, \Omega\right)$ satisfy

$$
W^{b 1}=W^{b}\left(x^{e}\left(d^{s}(\beta, \Omega), 1, \Omega\right), d^{s}(\beta, \Omega)\right)=p^{b}
$$

and

$$
W^{s 1}=W^{s}\left(y^{e}\left(d^{b}(\alpha, \Omega), 1, \Omega\right), d^{b}(\alpha, \Omega)\right)=p^{s} .
$$

Then with Equations (3-7), Equations (2) can be restated as Proposition 1.

Proposition 1. The optimal trajectory $\left(\tilde{p}^{b}, \tilde{p}^{s}, \tilde{x}, \tilde{y}\right)$ should satisfy

$$
\frac{p^{b}-[\overbrace{c_{x}-e^{\delta t} \dot{\lambda}^{1}}^{\text {marginal costs }}-\overbrace{y W_{2}^{s e}}^{\text {network benefits }}-\overbrace{\left(\Delta W_{1}^{b} \theta^{2} G_{p^{b}}^{s}+\Delta W_{2}^{s} \theta^{1} G_{p^{s}}^{b}\right)}^{\text {the effect of expectaion bias }}]}{p^{b}}=\frac{1}{\underbrace{\varepsilon^{b}}_{\text {price elasticity }}}
$$

and

$$
\frac{p^{s}-[\overbrace{c_{y}-e^{\delta t} \dot{\lambda}^{2}}^{\text {marginal costs }}-\overbrace{x W_{2}^{b e}}^{\text {network benefits }}-\overbrace{\left(\Delta W_{1}^{s} \theta^{1} G_{p^{s}}^{b}+\Delta W_{2}^{b} \theta^{2} G_{p^{b}}^{s}\right)}^{\text {the effect of expectaion bias }}]}{p^{s}}=\frac{1}{\underbrace{\varepsilon^{s}}_{\text {price elasticity }}},
$$

where $\Delta W_{i}^{b}=W_{i}^{b e}-W_{i}^{b 1}, \Delta W_{i}^{s}=W_{i}^{s e}-W_{i}^{s 1}, i=1,2, \varepsilon^{b}=\frac{-p^{b}}{x W_{1}^{b e}}$ and $\varepsilon^{s}=\frac{-p^{s}}{y W_{1}^{s e}}$.

Similar to the Lerner formula in Armstrong (2006), Proposition 1 shows that the optimal prices at any time are also affected by the marginal costs, network benefits and price elasticity. However, the economic meanings of them are changed. Take buyers as an example. From Equations (1-2), we can prove that $\lambda^{1}(\tau)=\Pi_{x(\tau)}^{\tau}$ and $\lambda^{2}(\tau)=\Pi_{y(\tau)}^{\tau}$, where $\Pi^{\tau}=\int_{\tau}^{+\infty} e^{-\delta t} \pi(t, \Omega) d t$. The marginal costs then also include the marginal loss from attracting one buyer $\left(-e^{\delta t} \lambda^{1}=\frac{d}{d t}\left(\frac{\partial\left(\Pi-\Pi^{t}\right)}{\partial x(t)}\right)\right)$ as increasing the number of buyers requires time. The price elasticity and network benefits should consider the existing numbers of users $\left(x\right.$ in $\varepsilon^{b}$ and $y$ in $\left.y W_{2}^{\text {se }}\right)$ 
Pricing in Two-Sided Markets with Dynamic Subscription Process and Users' Expectations

rather than the demands of users. In addition, the platform should consider the effect of expectation bias that comes from the difference between beliefs and reality as well. However, in the final equilibrium, such effect does not exist since $\Delta W_{i}^{b}=\Delta W_{i}^{s}=0, i=1,2$.

\section{Equilibrium analysis}

In the steady-state equilibrium denoted by $\Omega^{*}=\left(p^{b^{*}}, p^{s^{*}}, x^{*}, y^{*}\right)$, we obtain that $\dot{x}=\dot{y}=\dot{p}^{b}=\dot{p}^{s}=\dot{\theta}^{1}=\dot{\theta}^{2}=0$ and that $W^{b}\left(x^{*}, y^{*}\right)=p^{b^{*}}$ and $W^{s}\left(y^{*}, x^{*}\right)=p^{s^{*}}$ due to Lemma 2. Hence in equilibrium, the prices are functions of $x$ and $y$, i.e.,

$$
p^{b}(x, y)=W^{b}(x, y) ; \quad p^{s}(x, y)=W^{s}(y, x),
$$

Proposition 2. In the equilibrium, the optimal prices should satisfy

$$
\frac{p^{b}-\left(c_{x}-y p_{x}^{s}+\delta \theta^{1}\right)}{p^{b}}=\frac{1}{\varepsilon^{b}} ; \quad \frac{p^{s}-\left(c_{y}-x p_{y}^{b}+\delta \theta^{2}\right)}{p^{s}}=\frac{1}{\varepsilon^{s}}
$$

where $\varepsilon^{b}=-p^{b} /\left(x p_{x}^{b}\right)$ and $\varepsilon^{s}=-p^{s} /\left(y p_{y}^{s}\right)$.

In the steady-state equilibrium, the above results show that, the platform owner does not need to consider the effect of expectation bias in equilibrium. It implies that even if users may have unreasonable high expectations in the developing stage of the platform, they will finally find the truth. The platform should not be over-optimistic. The real status of the platform, such as the service quality and the numbers of users, is the key to make profits. In addition, the results also imply that even the willingness to pay functions and the cost functions are symmetric, the optimal prices on the two sides may be different due to the existence of $\delta \theta^{1}$ and $\delta \theta^{2}$. Therefore it gives rise to another explanation about skewed pricing in two-sided markets since the growth rates and the weight coefficients of the two sides also affect the optimal prices.

Next, we study the effect of some exogenous parameters. From Equations (11), we obtain that the equilibrium satisfies

and

$$
\varphi^{1}(x, y)=p^{b}(x, y)-c_{x}+y p_{x}^{s}+x p_{x}^{b}-\delta \theta^{1}=0
$$

$$
\varphi^{2}(x, y)=p^{s}(x, y)-c_{y}+x p_{y}^{b}+y p_{y}^{s}-\delta \theta^{2}=0
$$

Let $t^{*}$ denote the time in equilibrium. As $\lambda^{1}(\tau)=\Pi_{x(\tau)}^{\tau}$ and $\lambda^{2}(\tau)=\Pi_{y(\tau)}^{\tau}$, we

obtain $\tilde{\Pi}\left(t^{*}, \Omega\right) \square \int_{0}^{x} \varphi^{1}(x, y)=\int_{0}^{y} \varphi^{2}(x, y)=\delta\left(\int_{t^{*}}^{+\infty} e^{-\delta\left(t-t^{*}\right)} \pi\left(t^{*}, \Omega\right) d t+e^{\delta t^{*}} \int_{0}^{t^{*}} e^{-\delta t} \pi(t, \Omega) d t\right)$. Hence, $\tilde{\Pi}\left(t^{*}, \Omega\right) / \delta$ denotes the total profits discounted at time $t^{*}$. We assume that $\tilde{\Pi}\left(t^{*}, \Omega\right)$ is a concave function of $x\left(t^{*}\right)$ and $y\left(t^{*}\right)$. Then we have $\varphi_{x}^{1}<0, \varphi_{y}^{2}<0$ and the determinant of $\Phi=\left[\begin{array}{cc}\varphi_{x}^{1} & \varphi_{y}^{1} \\ \varphi_{x}^{2} & \varphi_{y}^{2}\end{array}\right]$ is positive, i.e., $\operatorname{det}(\Phi)>0$. 
Yang Geng, Yu-Lin Zhang

According to the theorem of implicit function, we then obtain

$$
\frac{\partial N}{\partial \zeta}=-\Phi^{-1}\left[\begin{array}{c}
\varphi_{\zeta}^{1} \\
\varphi_{\zeta}^{2}
\end{array}\right]=\frac{-1}{\operatorname{det}(\Phi)}\left[\begin{array}{cc}
\varphi_{y}^{2} & -\varphi_{y}^{1} \\
-\varphi_{x}^{2} & \varphi_{x}^{1}
\end{array}\right]\left[\begin{array}{l}
\varphi_{\zeta}^{1} \\
\varphi_{\zeta}^{2}
\end{array}\right]
$$

from Equations (12-13), where $N=\left[x^{*}, y^{*}\right]^{T}$ and $\zeta$ is an exogenous parameter.

Proposition 3. The equilibrium numbers of users satisfy $x_{G^{b^{b}}}^{*}>0$ and $y_{G^{s}}^{*}>0$.

Proposition 3 shows that if the growth rate of users on one side is high, the platform should attract more users on this side. The reason is that in a dynamic framework, if the number of users on one side grows rapidly, the costs of attracting users on this side will be low.

Proposition 4. In the equilibrium, the optimal numbers of users satisfy

$$
\operatorname{sgn}\left\{x_{\alpha}^{*}\right\}=\operatorname{sgn}\left\{\theta_{\alpha}^{1} / \theta_{\alpha}^{2}-\varphi_{y}^{1} / \varphi_{y}^{2}\right\} ; \quad \operatorname{sgn}\left\{y_{\alpha}^{*}\right\}=\operatorname{sgn}\left\{\theta_{\alpha}^{2} / \theta_{\alpha}^{1}-\varphi_{x}^{2} / \varphi_{x}^{1}\right\} .
$$

In addition, if $x_{\alpha}^{*}<0, y_{\alpha}^{*}>0$, and vice versa. In the completely symmetric case, we have $p_{\alpha}^{b^{*}}=p_{\alpha}^{s^{*}}<0$ and $x_{\alpha}^{*}=y_{\alpha}^{*}>0$.

Unlike the results in Proposition 3, the above results show that the effect of $\alpha$ on the number of buyers is uncertain. This uncertainty derives from the tradeoff between two benefits. First, with increasing $\alpha$, the number of buyers becomes more valuable as it affects the subscription decisions of buyers. Second, it is also profitable to attract more sellers by lowing their prices because the prices on the sellers' side will affect the expectations of buyers. Proposition 4 also implies that with increasing $\alpha$, the platform owner should attract more users on at least one side. The reason is that if we exclude the tradeoff between the two sides, i.e., the completely symmetric case, with the increasing of the weight coefficients, the platform owner should set lower prices and attract more users since users become more sensitive to the prices.

Proposition 5. In the equilibrium, the optimal numbers of users satisfy

$$
\operatorname{sgn}\left\{x_{\delta}^{*}\right\}=\operatorname{sgn}\left\{\varphi_{y}^{1} / \varphi_{y}^{2}-\theta^{1} / \theta^{2}\right\} ; \quad \operatorname{sgn}\left\{y_{\delta}^{*}\right\}=\operatorname{sgn}\left\{\varphi_{x}^{2} / \varphi_{x}^{1}-\theta^{2} / \theta^{1}\right\} .
$$

In addition, if $x_{\delta}^{*}>0, y_{\delta}^{*}<0$, and vice versa. In the completely symmetric case, we have $p_{\delta}^{b^{*}}=p_{\delta}^{s^{*}}>0$ and $x_{\delta}^{*}=y_{\delta}^{*}<0$.

Differing from the results in Proposition 4, if the discount rate is high, the platform bears high costs of attracting users when using a low-price strategy. Hence, in the completely symmetric case, the platform should set high prices and attract less users when the discount rate is high. Similarly, in the asymmetric case where the tradeoff between the two sides is important, with the increasing of the discount rate, the above result shows that the platform should attract less users on at least one side.

\section{Numerical Analysis}

In this section, we illustrate our results based on Evans and Schmalensee (2010) who study the dynamic subscription process in two-sided markets. 
Pricing in Two-Sided Markets with Dynamic Subscription Process and Users' Expectations

Specifically, the willingness-to-pay functions of buyers and sellers are

$$
W^{b}\left(\xi, y^{e}\right)=e^{b}(1-\xi) y^{e} ; W^{s}\left(\eta, x^{e}\right)=e^{s}(1-\eta) x^{e}
$$

The growth rate functions are linear, i.e.,

$$
\dot{x}=v^{b}\left[d^{b}(\alpha, \Omega)-x\right] ; \dot{y}=v^{s}\left[d^{s}(\beta, \Omega)-y\right]
$$

where the initial numbers of users are zeroes: $x(0)=0$ and $y(0)=0$. Besides, we also assume that the cost function of the platform is linear, i.e., $c(x, y)=c^{b} x+c^{s} y$.

We first analyze the case where users on the two sides are completely myopic, i.e., $\alpha=\beta=0$. Since decreasing the numbers of users may send a negative signal to users, we assume that $0 \leq p^{b} \leq W^{b}(x, y)$ and $0 \leq p^{s} \leq W^{s}(y, x)$.

We then have the current value Lagrangian

$$
L^{c}=H^{c}+\zeta^{b}\left(W^{b}(x, y)-p^{b}\right)+\zeta^{s}\left(W^{s}(y, x)-p^{s}\right)+\psi^{b} p^{b}+\psi^{s} p^{s} .
$$
follows

By the maximum principle, we obtain the optimal bang-bang pricing as

$$
\tilde{p}^{i}(t)=\left\{\begin{array}{cc}
\bar{W}^{i} & H_{p^{i}}^{c}>0 \\
0 & H_{p^{i}}^{c}<0
\end{array} \quad i=b, s,\right.
$$

where $\bar{W}^{b}=e^{b}(1-x(t)) y(t)$ and $\bar{W}^{s}=e^{s}(1-y(t)) x(t)$.

From Equation (16), we obtain that the number of users on side $i$ will grow when $\tilde{p}^{i}(t)=0$ and keep constant when $\tilde{p}^{i}(t)=\bar{W}^{i}$. With Equations (15), we then prove that the shortest length of time that the number of buyers (resp. sellers) grows from 0 to $x^{*}$ (resp. $\left.y^{*}\right)$, denoted by $t^{x}$ (resp. $\left.t^{y}\right)$ satisfies $x^{*}=1-e^{-v^{b} t^{*}}$ (resp. $y^{*}=1-e^{-v^{s} t^{y}}$ ). Since the profits in equilibrium are much higher than the profits in non-equilibrium, we study a simple case where the platform will not delay the time between non-equilibrium and equilibrium $\left(T^{*}\right)$, which implies that $T^{*}=\max \left(t^{x}, t^{y}\right)$. 
Yang Geng, Yu-Lin Zhang
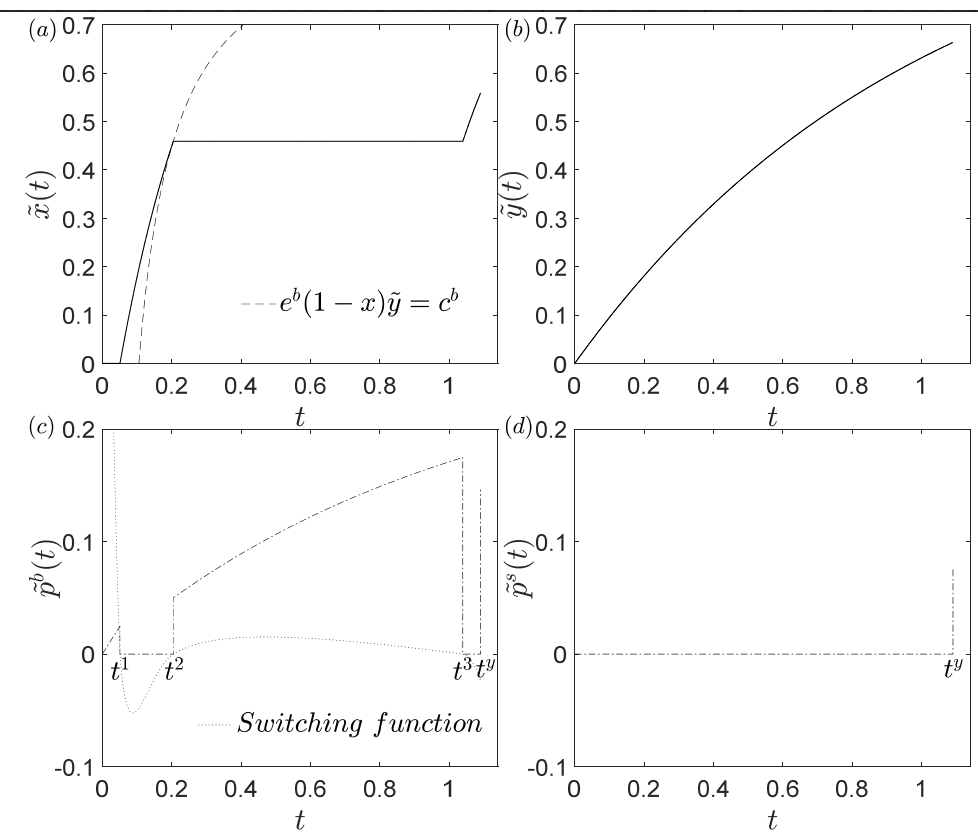

Figure 2. Optimal trajectory in the myopic case ${ }^{2}$

Figure 2 shows an example of the myopic case. In Fig. 2 (c) and (d), there are four switching times $\left(t^{1}, t^{2}, t^{3}\right.$ and $\left.t^{y}\right)$ on the buyers' side and one switching time $\left(t^{y}\right)$ on the sellers' side. At each time, the platform changes its pricing strategies.

In the first time interval $\left(t \in\left[0, t^{1}\right)\right)$, we can see that in the optimal trajectory, the platform owner delays the entrance of buyers and attracts sellers first $(\tilde{x}(t)=0$, see Fig. 2 (a)). The reason is that when the number of sellers is too small, the costs of attracting a large user base on the buyers' side are large. Hence, as shown in Fig. 3 (a, c-d), when $c^{b}, v^{b}$ or $\delta$ is high, the platform owner should delay the entrance time of buyers $\left(t^{1}\right)$. Similarly, if the benefits of attracting buyers are small, e.g., $e^{b}$ or $v^{s}$ is low, we obtain the same results (see Fig. 3 (b-c)).

When $t \in\left[t^{1}, t^{2}\right)$, the optimal pricing strategy is $\tilde{p}^{b}(t)=0$. In this time interval, the platform attracts buyers through subsidies (below marginal costs). The problem for the platform is when to stop subsidies. In a simple case with $\delta=0$, Fig. 2 (a) shows that the platform should stop subsidies if the marginal buyer's willingness-to-pay equals the marginal cost of attracting buyers $\left(e^{b}\left(1-\tilde{x}\left(t^{2}\right)\right) \tilde{y}\left(t^{2}\right)=c^{b}\right) .{ }^{3}$ The reason is that when $\tilde{p}^{b}\left(t^{2}\right)=e^{b}\left(1-\tilde{x}\left(t^{2}\right)\right) \tilde{y}\left(t^{2}\right)<c^{b}$

2 The parameters used are $e^{b}=0.5, e^{s}=0.4, c^{b}=c^{s}=0.05, v^{b}=4, v^{s}=1$ and $\delta=0$.

${ }^{3}$ At time $t^{1}$, we have $\theta^{1}\left(t^{1}\right)=0$ since $H_{p^{b}}^{c}=x\left(t^{1}\right)-\theta^{1}\left(t^{1}\right) v^{b} /\left(e^{b} y\left(t^{1}\right)\right)=0$ and $x\left(t^{1}\right)=0$. When 
Pricing in Two-Sided Markets with Dynamic Subscription Process and Users' Expectations

$\overline{\left(\text { resp., } \quad \tilde{p}^{b}\left(t^{2}\right)=e^{b}\left(1-\tilde{x}\left(t^{2}\right)\right) \tilde{y}\left(t^{2}\right)>c^{b}\right) \text {, it is more profitable to delay (resp., advance) }}$ the time interval $\left(t^{1}, t^{2}\right)$. Besides, in the case with $\delta>0$, Fig. 3 (d) shows that $\tilde{p}^{b}\left(t^{2}\right)=e^{b}\left(1-\tilde{x}\left(t^{2}\right)\right) \tilde{y}\left(t^{2}\right)>c^{b}$. In this case, as $\delta>0$, delaying the time interval $\left(t^{1}, t^{2}\right)$ can reduce the loss from subsidies.
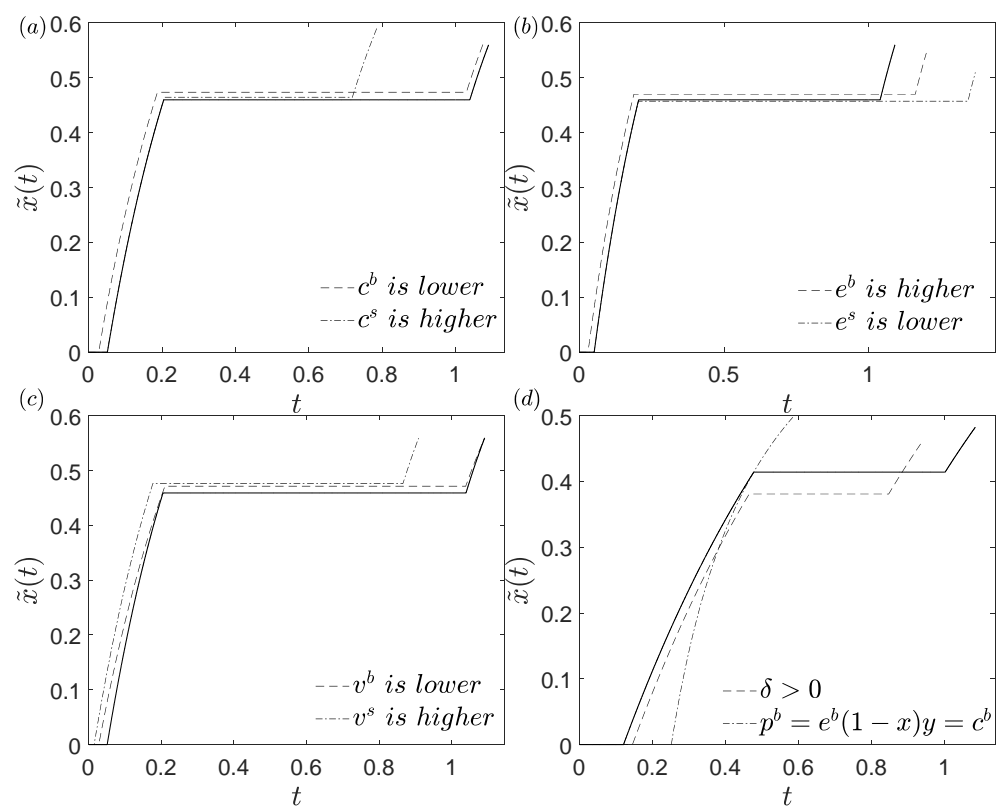

Figure 3. Comparison between different parameters ${ }^{4}$

In the third and fourth time intervals, we see that the number of buyers remains unchanged when $t \in\left[t^{2}, t^{3}\right)$ and increases again when $t \in\left[t^{3}, t^{y}\right]$. Hence, except for the growth of users on the two sides, the platform should also attach importance to the proportion between the existing numbers of users on the two sides.

Next, we study a symmetric case with $\alpha, \beta>0$. In this case, as shown in Fig.

$t \in\left(t^{1}, t^{2}\right)$, we obtain $\dot{\theta}^{1}=c^{b}+v^{b} \theta^{1}$. Then we can prove that $\theta^{1}\left(t^{2}\right)=c^{b} x\left(t^{2}\right) /\left(v^{b}\left(1-x\left(t^{2}\right)\right)\right)$. Finally, because $y\left(t^{2}\right)=\theta^{1} v^{b} /\left(e^{b} x\left(t^{2}\right)\right)$, we obtain that $\tilde{p}^{b}\left(t^{2}\right)=e^{b}\left(1-\tilde{x}\left(t^{2}\right)\right) \tilde{y}\left(t^{2}\right)=c^{b}$.

4 The parameters of the basic case (the solid line) in Fig. 3 (a), (b) and (c) are the same as those in Fig. 2. In Fig. 3 (a), the lower $c^{b}$ is 0.045 and the higher $c^{s}$ is 0.1 . In Fig. 3 (b), the lower $e^{s}$ is 0.3 and the higher $e^{b}$ is 0.55 . In Fig. 3 (c), the lower $v^{b}$ is 0.35 and the higher $v^{s}$ is 0.12 . In Fig. 3 (d), the parameters of the basic case (the solid line) are $e^{b}=0.45, e^{s}=0.45, c^{b}=0.1, c^{s}=0.05$, $\delta=0, v^{b}=1.5$ and $v^{s}=1$. In addition, in the case where $\delta>0, \delta=0.2$. 
4, we see that the optimal prices cannot be higher than the boundary $W^{b}(x, x)=p^{b}$ (see curve $A B$ in Fig. 4 (a)) since when $W^{b}(x, x)<p^{b}$, the demand of buyers will be zero, i.e., $d^{b}(\alpha, \Omega)=0$, and of course, such strategy is infeasible.
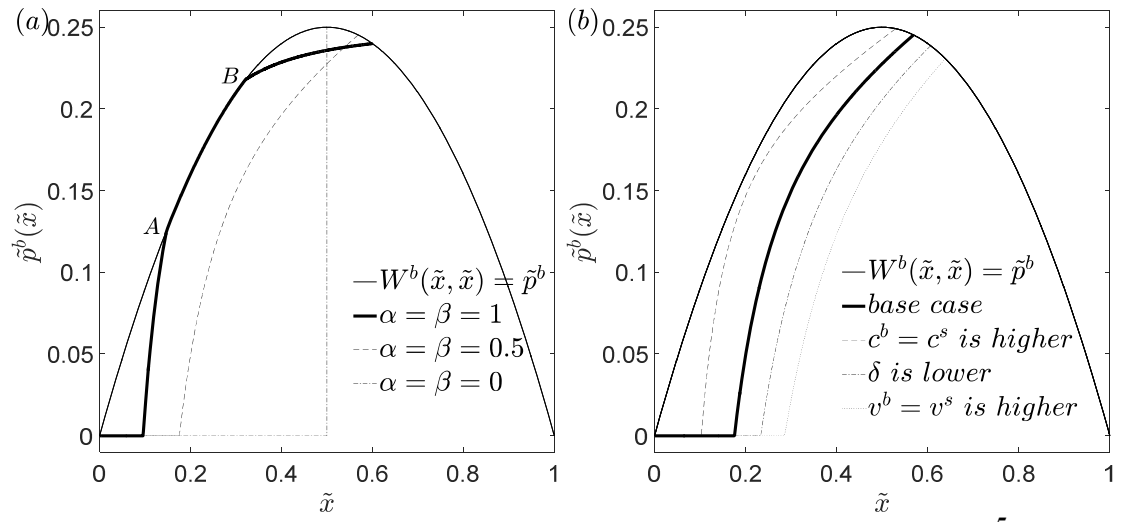

Figure 4. Optimal trajectories in the symmetric case $\mathrm{e}^{5}$

In addition, when $\alpha$ and $\beta$ are high, 1) in non-equilibrium, the platform owner needs to attract users on both sides as users are also sensitive to the number of users on the same side, which increases the costs of attracting users; and 2) in equilibrium, since users are more sensitive to the prices on the other side, a low-price strategy becomes profitable. Hence if $\alpha$ and $\beta$ are high, the price trajectories increase rapidly in the period when the numbers of users are low and then converge to low equilibrium prices. Similarly, when $\delta$ or $c^{b}=c^{s}$ is low or $v^{b}=v^{s}$ is high, the price trajectories increase rapidly in the period when the numbers of users are high (the costs of attracting users are low) and then converge to low equilibrium prices (a low-price strategy becomes profitable).

\section{Conclusions}

In this paper, we study dynamic pricing in two-sided markets while considering users' expectations. The theoretical results show that similar to the static framework, the optimal prices at any time are affected by the marginal costs, network benefits and price elasticity. The difference is that the marginal costs in the dynamic model should include the loss from attracting users as increasing the numbers of users requires time, and the network benefits and price elasticity depend on the existing numbers of users rather than on the demands of users. In addition, the expectation

${ }^{5}$ Other parameters in Figure 4 (a) are $e^{b}=e^{s}=1, c^{b}=c^{s}=0, v^{b}=v^{s}=0.1$ and $\delta=0.1$. The parameters in the base case of Fig. 4 (b) are the same as the case with $\alpha=\beta=0.5$ in Fig. 4 (a). In addition, in Fig. 4 (b), the higher $c^{b}$ and $v^{b}$ are 0.1 and 0.5 , respectively, and the lower $\delta$ is 0.05 . 
Pricing in Two-Sided Markets with Dynamic Subscription Process and Users' Expectations

bias that comes from the difference between beliefs and reality affects the optimal prices in non-equilibrium. However, it does not affect the equilibrium prices as users are rational. Hence, in the long term, the real status of the platform, such as the service quality and the numbers of users, is the key to make profits.

In the steady-state equilibrium, we find that in the dynamic framework, except for the indirect network effects, the growth rates and users' beliefs may also give rise to skewed pricing. When the discount rate is low or when the weight coefficients of the conjectured numbers that depend on users' beliefs are high, in the symmetric case, the platform should set low prices to attract more users on the two sides, while in the asymmetric case where the tradeoff between the two sides is important, the platform should attract more users on at least one side.

The numerical results show that in the early period, the platform may delay the entrance of users on one side and attract only users on the other side if the costs of attracting a large user base on this side are large. Hence, except for the growth of users, the platform should attach importance to the proportion between the numbers of users on the two sides as well. In addition, if the weight coefficients are high, the optimal price trajectories increase rapidly in the period when the numbers of users are low and then converge to low equilibrium prices.

\section{ACKNOWLEDGEMENTS}

This work is supported by the National Natural Science Foundation of China (72071040, 71671036), the major project of philosophy and social science research in colleges and universities in Jiangsu province (2018SJZDA005) and Ministry of Education in China Project of Humanities and Social Sciences (20YJC630142).

\section{Appendix}

\section{Proof of Lemma 1}

When $\bar{\xi} \in\left\{\bar{\xi}: y^{e}(\bar{\xi}, 1, \Omega)>0\right\}$, from (A3) and $\bar{\xi}=x$, we have $W^{s}\left(y^{e 1}, \bar{\xi}\right)=p^{s}$. Then we obtain $y_{\bar{\xi}}^{e l}>0$ as $W_{1}^{s}<0$ and $W_{2}^{s}>0$. In addition, since $W^{s}\left(y^{e 1}, \bar{\xi}\right)$ is concave in $\left(y^{e 1}, \bar{\xi}\right)$ and $W_{12}^{s} \leq 0$, we can prove that $y_{\overline{\xi \xi}}^{e 1}>0$. Finally, according to the concavity of $W^{b}\left(\bar{\xi}, y^{e}(\bar{\xi}, \alpha, \Omega)\right)$ with respect to $\left(\bar{\xi}, y^{e}(\bar{\xi}, \alpha, \Omega)\right)$ and the results obtained above, we obtain that $W_{\overline{\xi \xi}}^{b}<0$. When $\bar{\xi} \in\left\{\bar{\xi}: \alpha y^{e}(\bar{\xi}, 1, \Omega)=0\right\}$, from (A3), we have $W_{\xi}^{b}=0$ if $\alpha=1$. When $\alpha \neq 1$, we have $W_{\xi}^{b}<0$ as $W_{1}^{b}<0$.

\section{Proof of Lemma 2}

In the equilibrium, due to the discussion in Section 3.2, we have $W^{b}(x, y)=p^{b}$ and $W^{s}(y, x)=p^{s}$ because $x^{e}(\bar{\eta}, \beta, \Omega)=x$ and $y^{e}(\bar{\xi}, \alpha, \Omega)=y$. In addition, when $W^{b}(x, y)=p^{b} \quad$ and $\quad W^{s}(y, x)=p^{s}$, we can prove that $x^{e}(\bar{\eta}, \beta, \Omega)=x$, 
Yang Geng, Yu-Lin Zhang

$y^{e}(\bar{\xi}, \alpha, \Omega)=y$ and $\dot{x}=0, \dot{y}=0$. Then we obtain Lemma 2.

Proof of Proposition 1

From Equations (4) and (7), we have $W_{1}^{b e} d_{p^{b}}^{b}(\alpha, \Omega)+W_{2}^{b e} \alpha y_{d^{b}}^{e} d_{p^{b}}^{b}(\alpha, \Omega)=1$ and $W_{1}^{s 1} y_{d^{b}}^{e}+W_{2}^{s 1}=0$. Simplifying these two equations, we obtain $d_{p^{b}}^{b}(\alpha, \Omega)=W_{1}^{s 1} / \Delta^{\alpha}$, where $\Delta^{\alpha}=W_{1}^{b e} W_{1}^{s 1}-\alpha W_{2}^{b e} W_{2}^{s 1}$. Similarly, from Equations (4-7), we can prove that $d_{p^{s}}^{b}=-\alpha W_{2}^{b e} / \Delta^{\alpha}, \quad d_{y}^{b}=-(1-\alpha) W_{2}^{b e} W_{1}^{s 1} / \Delta^{\alpha}, d_{p^{b}}^{s}=-\beta W_{2}^{s e} / \Delta^{\beta}, d_{p^{s}}^{s}=W_{1}^{b 1} / \Delta^{\beta}$ and $d_{x}^{s}=-(1-\beta) W_{2}^{s e} W_{1}^{b 1} / \Delta^{\beta}$, where $\Delta^{\beta}=W_{1}^{b 1} W_{1}^{s e}-\beta W_{2}^{b 1} W_{2}^{s e}$.

Substituting the Equations above into Equations (3), we obtain

$$
\theta^{1}=\frac{\left(-x W_{1}^{b 1}-\beta y W_{2}^{s e}\right) \Delta^{\alpha}}{G^{b^{\prime}} \Delta^{\alpha \beta}} ; \theta^{2}=\frac{\left(-y W_{1}^{s 1}-\alpha x W_{2}^{b e}\right) \Delta^{\beta}}{G^{s^{\prime}} \Delta^{\alpha \beta}}
$$

where $\Delta^{\alpha \beta}=W_{1}^{b 1} W_{1}^{s 1}-\alpha \beta W_{2}^{b e} W_{2}^{s e}$. With Equations (17), Equations (2) can then be restated as Equations (8-9).

\section{Proof of Proposition 2}

In the steady-state equilibrium, from Equations (4-7), we have $\Delta W_{i}^{b}=\Delta W_{i}^{s}=0, i=1,2$, and from Equations (10), we obtain that $p_{x}^{b}=W_{1}^{b e}$, $p_{x}^{s}=W_{2}^{s e}, p_{y}^{b}=W_{2}^{b e}$ and $p_{y}^{s}=W_{1}^{s e}$. Besides, since $\theta^{i}=e^{\delta t} \lambda^{i}$ and $\dot{\theta}^{i}=0$, we have $e^{\delta t} \dot{\lambda}^{i}=-\delta \theta^{i}+\dot{\theta}^{i}=-\delta \theta^{i}, \quad i=1,2$. Substituting these results into Proposition 1, we have Equations (11).

\section{Proof of Proposition 3, 4, 5}

In the equilibrium, from Equations (17), we have

$$
\theta^{1}=\frac{\left(-x p_{x}^{b}-\beta y p_{x}^{s}\right)\left(p_{x}^{b} p_{y}^{s}-\alpha p_{y}^{b} p_{x}^{s}\right)}{G^{b^{\prime}}\left(p_{x}^{b} p_{y}^{s}-\alpha \beta p_{y}^{b} p_{x}^{s}\right)} ; \theta^{2}=\frac{\left(-y p_{y}^{s}-\alpha x p_{y}^{b}\right)\left(p_{x}^{b} p_{y}^{s}-\beta p_{y}^{b} p_{x}^{s}\right)}{G^{s^{\prime}}\left(p_{x}^{b} p_{y}^{s}-\alpha \beta p_{y}^{b} p_{x}^{s}\right)}
$$

where $G^{b^{\prime}}=G^{b^{\prime}}(0)$ and $G^{s^{\prime}}=G^{s^{\prime}}(0)$ since $d^{b}(\alpha, \Omega)=x$ and $d^{s}(\beta, \Omega)=y$.

(1) For $\zeta=G^{b^{\prime}}(0)$ or $\zeta=G^{s^{\prime}}(0)$, from Equations (12-13), we have $\varphi_{\zeta}^{i}=-\delta \theta_{\zeta}^{i}$, and from Equations (18), we have $\theta_{G^{s^{s}}}^{1}=\theta_{G^{b}}^{2}=0, \theta_{G^{b}}^{1}<0$ and $\theta_{G^{s}}^{2}<0 .{ }^{6}$ Then with Equation (14), $\varphi_{x}^{1}<0$ and $\varphi_{y}^{2}<0$, we prove Proposition 3.

(2) For $\zeta=\alpha$, from Equations (12-13), we obtain $\varphi_{\zeta}^{i}=-\delta \theta_{\zeta}^{i}$, and from Equations (18), we obtain

$$
\theta_{\alpha}^{1}=\frac{\left(-x p_{x}^{b}-\beta y p_{x}^{s}\right)(\beta-1) p_{x}^{b} p_{y}^{s} p_{y}^{b} p_{x}^{s}}{G^{b^{\prime}}\left(p_{x}^{b} p_{y}^{s}-\alpha \beta p_{y}^{b} p_{x}^{s}\right)^{2}} ; \theta_{\alpha}^{2}=\frac{p_{y}^{s} p_{y}^{b}\left(-x p_{x}^{b}-\beta y p_{x}^{s}\right)\left(p_{x}^{b} p_{y}^{s}-\beta p_{y}^{b} p_{x}^{s}\right)}{G^{s^{\prime}}\left(p_{x}^{b} p_{y}^{s}-\alpha \beta p_{y}^{b} p_{x}^{s}\right)^{2}} .
$$

In the steady-state equilibrium, since

$$
d W^{b}(x, y)=p_{x}^{b} d x+p_{y}^{b} d y=d x\left(p_{x}^{b}+p_{y}^{b}(d y / d x)\right)=d x\left(p_{x}^{b}-p_{y}^{b} p_{x}^{s} / p_{y}^{s}\right),
$$

\footnotetext{
${ }^{6}$ Due to the economic meaning of $\theta^{1}$ and $\theta^{2}$, we consider that the two costate variables are positive.
} 
Pricing in Two-Sided Markets with Dynamic Subscription Process and Users' Expectations

we assume $p_{x}^{b}-p_{y}^{b} p_{x}^{s} / p_{y}^{s}<0$ to make sure that the equilibrium is stable. We then have $p_{x}^{b} p_{y}^{s}-\alpha \beta p_{y}^{b} p_{x}^{s}>0$ and $\theta_{\alpha}^{i}<0, i=1,2$. Finally from Equation (14), $\varphi_{x}^{1}<0$ and $\varphi_{y}^{2}<0$, we prove the general results in Proposition 4. In the symmetric case, we also have $\theta_{\alpha}^{1}=\theta_{\alpha}^{2}<0$. With $\operatorname{det}(\Phi)>0$, we obtain $\left|\varphi_{x}^{1}\right|=\left|\varphi_{y}^{2}\right|>\left|\varphi_{y}^{1}\right|=\left|\varphi_{x}^{2}\right|$. Then from Equation (14), we prove $x_{\alpha}^{*}=y_{\alpha}^{*}>0$. Besides, since $p_{x}^{b}-p_{y}^{b} p_{x}^{s} / p_{y}^{s}<0$, we obtain that $p_{x}^{b}+p_{y}^{b}<0$ and that $p_{\alpha}^{b^{*}}=p_{x}^{b^{*}} x_{\alpha}^{*}+p_{y}^{b^{*}} y_{\alpha}^{*}=\left(p_{x}^{b^{*}}+p_{y}^{b^{*}}\right) x_{\alpha}^{*}<0$.

(3) For $\zeta=\delta$, from Equations (12-13), we have $\varphi_{\zeta}^{i}=-\theta^{i}<0$. Then, similar to the proof in (2) above, we can prove Proposition 5.

\section{REFERENCES}

[1] Armstrong, M. (2006), Competition in Two-sided Markets. Rand Journal of Economics, 37(3), 668-691;

[2] Belleflamme, P. \& Peitz, M. (2019), Price Disclosure by Two-sided Platforms. International Journal of Industrial Organization, 67, 1-19;

[3] Cabral, L. (2011), Dynamic Price Competition with Network Effects. Review of Economic Studies, 78(1), 83-111;

[4] Cabral, L. (2019), Towards a Theory of Platform Dynamics. Journal of Economics \& Management Strategy, 28(1), 60-72;

[5] Caillaud, B. \& Jullien, B. (2003), Chicken \& Egg: Competition among Intermediation Service Providers. Rand Journal of Economics, 34(2), 309-328;

[6] Chen, K. \& Tse, E. T. (2008), Dynamic Platform Competition in Two-sided Markets. Working paper, Stanford University;

[7] Dhebar, A. \& Oren, S. S. (1985), Optimal Dynamic Pricing for Expanding Networks. Marketing Science, 4(4), 336-351;

[8] Dhebar, A. \& Oren, S. S. (1986), Dynamic Nonlinear Pricing in Networks with Interdependent Demand. Operations Research, 34(3), 384-394;

[9] Evans, D. S. \& Schmalensee, R. (2010), Failure to Launch: Critical Mass in Platform Businesses. Review of Network Economics, 9(4), 1-26;

[10] Gabszewicz, J. J. \& Wauthy, X. Y. (2014), Vertical Product Differentiation and Two-sided Markets. Economics Letters, 123(1), 58-61;

[11] Hagiu, A. \& Halaburda, H. (2014), Information and Two-sided Platform Profits. International Journal of Industrial Organization, 34, 25-35;

[12] Halaburda, H., Jullien, B. \& Yehezkel, Y. (2020), Dynamic Competition with Network Externalities: Why History Matters. Rand Journal of Economics, 51(1), 3-31;

[13] Halaburda, H. \& Yehezkel, Y. (2019), Focality Advantage in Platform

Competition. Journal of Economics \& Management Strategy, 28(1), 49-59; 
Yang Geng, Yu-Lin Zhang

[14] Jullien, B. \& Pavan, A. (2019), Information Management and Pricing in Platform Markets. Review of Economic Studies, 86(4), 1666-1703;

[15] Katz, M. L. \& Shapiro, C. (1985), Network Externalities, Competition, and Compatibility. American Economic Review, 75(3), 424-440;

[16] Markovich, S. \& Yehezkel, Y. (2019), Group Hug: Platform Competition with User-groups. Working paper, Northwestern University;

[17] Rochet, J.-C. \& Tirole, J. (2003), Platform Competition in Two-sided

Markets. Journal of the European Economic Association, 1(4), 990-1029;

[18] Rohlfs, J. (1974), A Theory of Interdependent Demand for a

Communications Service. Bell Journal of Economics and Management Science,

5(1), 16-37;

[19] Weyl, E. G. (2010), A Price Theory of Multi-sided Platforms. American

Economic Review, 100(4), 1642-1672. 